\title{
Core-Level Binding Energy Reveals Hydrogen Bonding Configurations of Water Adsorbed on $\mathrm{TiO}_{2}(110)$ Surface
}

\author{
C. Kamalø ${ }^{1,2,3,{ }^{*}}$ Nader Stenberg $\odot,{ }^{1}$ Lars Erik Walle $\odot,{ }^{4}$ Davide Ragazzon, ${ }^{5}$ Anne Borg, ${ }^{6}$ Per Uvdal $\odot,{ }^{7}$ \\ Natalia V. Skorodumova, ${ }^{5,8}$ Michael Odelius $\odot,{ }^{1, \dagger}$ and Anders Sandell $\oplus^{5, \$}$ \\ ${ }^{1}$ Department of Physics, Stockholm University, AlbaNova University Center, SE-106 91 Stockholm, Sweden \\ ${ }^{2}$ Theory and Simulations Laboratory, HRDS, Raja Ramanna Centre for Advanced Technology, Indore-452013, India \\ ${ }^{3}$ Homi Bhabha National Institute, Training School Complex, Anushakti Nagar, Mumbai-400094, India \\ ${ }^{4}$ Formation Physics, Petroleum Department, SINTEF Industry, NO-7465 Trondheim, Norway \\ ${ }^{5}$ Department of Physics and Astronomy, Uppsala University, Box 516, SE-751 20 Uppsala, Sweden \\ ${ }^{6}$ Department of Physics, NTNU-Norwegian University of Science and Technology, NO-7491 Trondheim, Norway \\ ${ }^{7}$ Chemical Physics, Department of Chemistry, Lund University, PO Box 124, SE-221 00 Lund, Sweden \\ ${ }^{8}$ Multiscale Materials Modelling, Department of Materials and Engineering, Royal Institute of Technology (KTH), \\ SE-10044, Stockholm, Sweden
}

(Received 9 September 2020; accepted 3 December 2020; published 7 January 2021)

\begin{abstract}
Using x-ray photoelectron spectroscopy of the oxygen $1 s$ core level, the ratio between intact $\left(\mathrm{D}_{2} \mathrm{O}\right)$ and dissociated (OD) water in the hydrated stoichiometric $\mathrm{TiO}_{2}(110)$ surface is determined at varying coverage and temperature. In the submonolayer regime, both the $\mathrm{D}_{2} \mathrm{O}$ :OD ratio and the core-level binding energy of $\mathrm{D}_{2} \mathrm{O}(\Delta \mathrm{BE})$ decrease with temperature. The observed variations in $\triangle \mathrm{BE}$ are shown with density functional theory to be governed crucially and solely by the local hydrogen bonding environment, revealing a generally applicable classification and details about adsorption motifs.
\end{abstract}

DOI: 10.1103/PhysRevLett.126.016102

How oxides wet has significant implications for materials science, (photo)catalysis, fuel cells, corrosion, and environmental remediation [1,2]. Most oxides are hydroxylated at room temperature and atmospheric vapor pressure. Wetting involving dissociation into adsorbed $\mathrm{H}$ and $\mathrm{OH}$ can be generated at perfect areas, edges, and defects [3-6]. The first water layer is particularly important, since it acts as a template for the growth of additional layers $[7,8]$. Among oxides, titanium dioxide $\left(\mathrm{TiO}_{2}\right)$ is an important material in the fields of photocatalysis, solar fuel cells, and self-cleaning coatings [3,4,9-12]. For molecularlevel understanding of hydration of oxide surfaces, the rutile $\mathrm{TiO}_{2}(110)$ single crystal surface $[3,4,12]$ is a carefully investigated model system. At ideal stoichiometry (denoted s- $\mathrm{TiO}_{2}$ ), it is entirely composed of alternating fivefold coordinated $\mathrm{Ti}$ ions $\left(\mathrm{Ti}_{5 c}\right)$ and twofold coordinated bridging oxygen ions $\left(\mathrm{O}_{b}\right)$. The dissociation on defect-free $\mathrm{TiO}_{2}(110)$ surface has been a controversial topic [13,14], but recent experimental and theoretical efforts are consistent with the fact that the first water layer on a defect-free surface is partially dissociated [15-21].

Published by the American Physical Society under the terms of the Creative Commons Attribution 4.0 International license. Further distribution of this work must maintain attribution to the author(s) and the published article's title, journal citation, and DOI.
A comprehensive molecular mechanism for the formation of the first water layer on the $\mathrm{s}-\mathrm{TiO}_{2}$ surface is still lacking. However, scanning tunneling microscope (STM) studies of water adsorbed on a reduced surface (i.e., with $\mathrm{O}_{b}$ vacancies) have revealed a clear propensity toward chain formation [22,23], and time lapse STM images have shown a temperature dependent diffusion along the $\mathrm{Ti}_{5 c}$ rows [24]. On a close to ideal $\mathrm{s}-\mathrm{TiO}_{2}$ surface, $\mathrm{x}$-ray photoelectron spectroscopy (XPS) in conjunction with density functional theory (DFT) and Monte Carlo simulations show a coverage dependent $\mathrm{OH}$ and $\mathrm{H}_{2} \mathrm{O}$ speciation, ranging from initial formation of stable hydroxyl pairs to predominantly molecular adsorption at increasing coverage [18]. A study combining molecular beam, STM, and ab initio molecular dynamics has shown that molecularly bound water is preferred over the surfacebound hydroxyls by only $0.035 \mathrm{eV}$ [25]. In conclusion, kinetic effects can clearly determine how the water layer is formed.

In this Letter, we demonstrate how detailed analysis of $\mathrm{O}$ $1 s$ XPS spectra reveals crucial information about the first water layer on the $\mathrm{TiO}_{2}(110)$ surface. The measurements are closely aligned with DFT based core-level binding energies (BE) to obtain a molecular-level understanding of the XPS signal for intact and dissociated water molecules in different hydrogen bonding configurations at various coverages. We show that XPS not only discloses the chemical state of adsorption but also probes the bonding 
configuration between the water molecules on the $\mathrm{TiO}_{2}(110)$ surface. Importantly, a general trend in the $\mathrm{BE}$ was discovered allowing for classification into three distinct classes of hydrogen donation and to determine their presence at different growth temperatures.

The photoemission measurements were performed at beam line D1011 at MAX-IV in Lund, Sweden, using a SES200 electron energy analyzer (SCIENTA) [26]. The O $1 s$ spectra were recorded in $60^{\circ}$ off normal emission using $610 \mathrm{eV}$ photons. The BE was referenced to the Fermi level of a $\mathrm{Pt}$ foil attached to the sample holder. The coverage is given in monolayers (ML, with $1 \mathrm{ML}$ being the density of $\mathrm{Ti}_{5 c}$ sites, $5.2 \times 10^{14} \mathrm{~cm}^{-2}$ ). We define the saturation uptake of water at $210 \mathrm{~K}$ to be $1 \mathrm{ML}$ [3,27]. The rutile $\mathrm{TiO}_{2}(110)$ single crystal (SurfaceNet $\mathrm{GmbH}$ ) was cleaned by sputtering $\left(\mathrm{Ar}^{+}, 1 \mathrm{keV}\right)$ and annealing to $900 \mathrm{~K}$ until no contaminations could be discerned with XPS. A TiO ${ }_{2}(110)$ surface with terraces having close to ideal stoichiometry was prepared at room temperature by exposing the sputtered and annealed surface first to water and then to $\mathrm{O}_{2}$ $[15,28-30]$. The low photon flux in combination with the extra precaution of using heavy water $\left(\mathrm{D}_{2} \mathrm{O}\right)$ effectively eliminates radiation induced effects [31,32].

The wetting of the $\mathrm{TiO}_{2}(110)$ surface was modeled, as in Ref. [18], using a periodic symmetric slab of $(2 \times 5)$ surface supercell with 7 O-Ti-O trilayers and a $15 \AA$ vacuum region above the surface [see Fig. 1(a)]. We consider different configurations with intact $(\mathbb{M})$ and dissociated $(\mathbb{D})$ water molecules adsorbed on the surface (at Ti adsorption sites), with coverages ranging from $10 \%$ to $100 \%$. We use the $\mathrm{CP} 2 \mathrm{~K}$ code to perform DFT calculations [33] using a van der Waals corrected Perdew-Burke-Ernzerhof (PBE) (a)

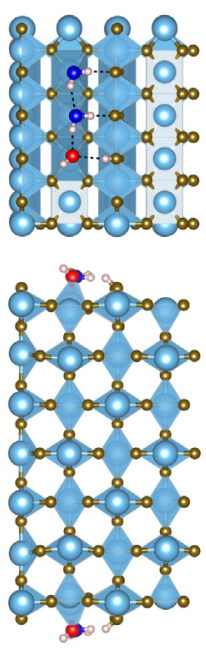

(b)

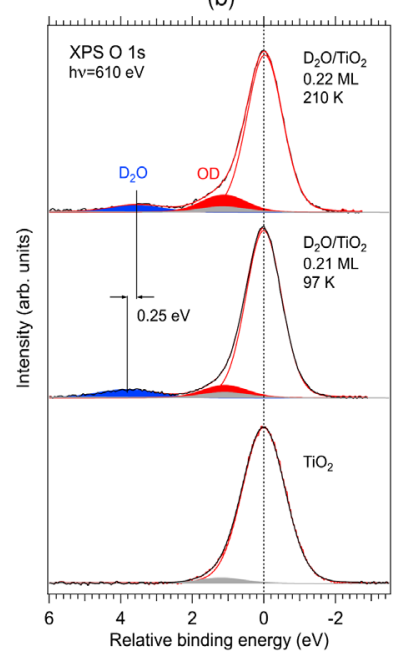

FIG. 1. (a) Structural model and (b) O $1 \mathrm{~s}$ spectra for water molecules adsorbed on $\mathrm{TiO}_{2}(110)$ surface, at two different temperatures, 210 and $97 \mathrm{~K}$, for an uptake of about $0.2 \mathrm{ML}$. The $\mathrm{BE}$ of $\mathrm{O} 1 s$ for the clean $\mathrm{s}-\mathrm{TiO}_{2}$ surface has been taken as reference. exchange-correlation (XC) functional [34,35]. The geometry optimizations have been performed using the Gaussian and plane wave method [36] with Goedecker-Teter-Hutter pseudopotentials, TZVP-MOLOPT basis sets, and an energy cutoff of $800 \mathrm{Ry}$. Core-level O $1 s$ BEs were calculated from total energy differences between the ground and core-ionized states, using an all-electron description on oxygen atoms (with pob-TZVP basis set) in the Gaussian augmented plane wave method [37]. The BE of an oxygen atom at the middle layer of the slab is used as reference.

Figure 1(b) shows $\mathrm{O} 1 \mathrm{~s}$ spectra at an uptake of $0.2 \mathrm{ML}$ at 97 and $210 \mathrm{~K}$, respectively, after background subtraction. Shown at the bottom for comparison is the $\mathrm{O} 1 s$ spectrum for the clean s- $\mathrm{TiO}_{2}$ surface. A relative $\Delta \mathrm{BE}$ is defined with respect to the component from $\mathrm{TiO}_{2}$. The curve fit of the spectrum (black line) for the clean surface is made using two components [red (strong) and gray (weak)] of which the weak feature is needed to capture the asymmetry of the peak toward the high BE side. This asymmetry is partly due to small amounts of OD left after the surface preparation $(\leq 0.03 \mathrm{ML})$ [15]. Upon adsorption and partial dissociation of water, two additional components, corresponding to $\mathrm{D}_{2} \mathrm{O}$ (blue) and OD (red), appear. We conclude that for 0.2 ML water, a higher degree of dissociation occurs at $210 \mathrm{~K}$ than at $97 \mathrm{~K}$. Further, $\triangle \mathrm{BE}$ for the peak associated with $\mathrm{D}_{2} \mathrm{O}$ is $0.25 \mathrm{eV}$ lower for deposition at $210 \mathrm{~K}$ than at $97 \mathrm{~K}$. The variation of the $\mathrm{O} 1 s \mathrm{BE}$ of $\mathrm{D}_{2} \mathrm{O}$ under different temperatures is central to learning about the adsorption and will be addressed comprehensively below. The partial overlap of the OD feature with the $\mathrm{TiO}_{2}$ peak prohibits detailed analysis of the OD species.

Figure 2 shows the results of real-time O $1 s$ XPS measurements upon water adsorption on the $\mathrm{s}-\mathrm{TiO}_{2}$ surface. The uptake series were conducted at three temperatures $\left(97,160\right.$, and $210 \mathrm{~K}$ ) keeping $3 \times 10^{-10}$ mbar $\mathrm{D}_{2} \mathrm{O}$ pressure. The $\mathrm{D}_{2} \mathrm{O}$ and OD coverages $(\Theta)$ are derived from the curve fitting described above. We also include the total water uptake, obtained as $\left[\Theta\left(\mathrm{D}_{2} \mathrm{O}\right)+0.5 \times \Theta(\mathrm{OD})\right]$ since $\mathrm{D}_{2} \mathrm{O}$ dissociation results in two OD groups. The uptake rate in all three cases is $0.042 \pm 0.004 \mathrm{ML} / \mathrm{min}$. At $97 \mathrm{~K}$, there

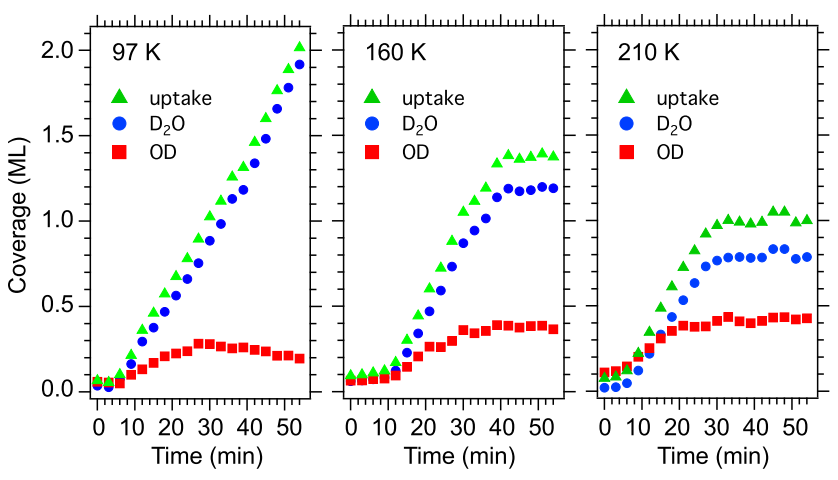

FIG. 2. Real-time monitoring of coverage upon water adsorption on $\mathrm{s}-\mathrm{TiO}_{2}$ surface at three temperatures, 97, 160, and $210 \mathrm{~K}$. 
(a)
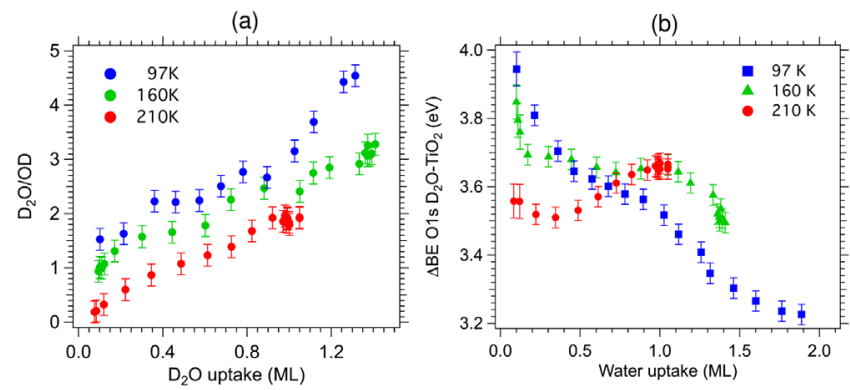

FIG. 3. (a) $\mathrm{D}_{2} \mathrm{O} / \mathrm{OD}$ ratio and (b) difference in $\mathrm{BE}(\Delta \mathrm{BE})$ of $\mathrm{O}$ $1 s$ peaks between $\mathrm{D}_{2} \mathrm{O}$ and $\mathrm{TiO}_{2}$ versus total coverage at three temperatures, 97, 160, and $210 \mathrm{~K}$.

is no limit in the $\mathrm{D}_{2} \mathrm{O}$ uptake since bulk ice is stable in UHV at this temperature. Water dissociation is still observed and the maximum OD coverage is $0.28 \pm$ $0.05 \mathrm{ML}$ (reached at $0.9 \mathrm{ML}$ uptake). At $160 \mathrm{~K}$, the saturation water uptake is $1.4 \mathrm{ML}$, partitioned into 1.2 ML molecular and 0.2 ML dissociated water. At $1 \mathrm{ML}$ uptake, the layer comprises $0.83 \pm 0.05 \mathrm{ML} \mathrm{D}_{2} \mathrm{O}$ and $0.35 \pm 0.05 \mathrm{ML}$ OD. Comparison to previous measurements at $210 \mathrm{~K}$ [18] shows a shift toward the intact form at the lower temperature.

Figure 3(a) presents the $\mathrm{D}_{2} \mathrm{O} / \mathrm{OD}$ ratio for each step in the growth series. All three series are characterized by a progressive increase of the $\mathrm{D}_{2} \mathrm{O} / \mathrm{OD}$ ratio as the coverage increases. Higher $\mathrm{D}_{2} \mathrm{O} / \mathrm{OD}$ ratios are apparent at lower temperature. The overall similar slopes in the submonolayer regime indicate that the reduced dissociation at lower temperature primarily stems from the behavior at very low coverage. The hydrogen-bonded configurations that form result from having a critical distance between dissociated water and a tendency for intact water to bond to already adsorbed species (nucleation versus aggregation) [18]. This effect can be expected to be more important at low coverage.

Our source of information regarding the water configurations formed is the $\mathrm{O} 1 s \mathrm{BE}$ of the $\mathrm{D}_{2} \mathrm{O}$ species. A redshift is known to occur upon hydrogen network formation when aggregates form $[7,38,39]$. Specifically, the variations in the $\mathrm{D}_{2} \mathrm{O} / \mathrm{OD}$ ratio entail changes in the $\mathrm{D}_{2} \mathrm{O}$ configuration, expected to translate into $\mathrm{O} 1 s \mathrm{BE}$ shifts. The $\mathrm{O} 1 s$ peak of $\mathrm{D}_{2} \mathrm{O}$ is well separated from the other two components, namely OD and $\mathrm{TiO}_{2}$, and its $\mathrm{BE}$ can be determined to within $\pm 30 \mathrm{meV}$ when the coverage is $>0.1 \mathrm{ML}$. Figure 3(b) shows the $\triangle \mathrm{BE}$ between the $\mathrm{O}$ $1 s$ peaks of $\mathrm{D}_{2} \mathrm{O}$ and $\mathrm{TiO}_{2}$ as a function of total amount of adsorbed water. At $97 \mathrm{~K}, \Delta \mathrm{BE}$ starts at a value of $3.95 \mathrm{eV}$ at $0.1 \mathrm{ML}$ uptake, after which it decreases progressively down to $3.2 \mathrm{eV}$ at $2 \mathrm{ML}$. At $160 \mathrm{~K}, \Delta \mathrm{BE}$ is first $3.92 \mathrm{eV}$ and then drops sharply to $3.69 \mathrm{eV}$ at about $0.2 \mathrm{ML}$. The $\Delta \mathrm{BE}$ value then stays constant at $3.67 \pm 0.02 \mathrm{eV}$ from 0.2 to $1 \mathrm{ML}$. Uptakes $>1 \mathrm{ML}$ lead to a decrease in $\triangle \mathrm{BE}$ due to the formation of the second layer, leading to a more extended hydrogen bonding network. In contrast, at $210 \mathrm{~K}, \Delta \mathrm{BE}$ starts at a low value $(3.56 \mathrm{eV})$, after which it drops even further (to $3.50 \mathrm{eV}$ ) at $0.3 \mathrm{ML}$ and then increases slowly, reaching $3.66 \mathrm{eV}$ at $1 \mathrm{ML}$, which is quite close to that observed at $160 \mathrm{~K}$. Interestingly, comparable $\mathrm{D}_{2} \mathrm{O} / \mathrm{OD}$ partitioning $(0.79 \mathrm{ML} / 0.42 \mathrm{ML}$ at $210 \mathrm{~K}$ and $0.83 \mathrm{ML} / 0.36 \mathrm{ML}$ at $160 \mathrm{~K}$ ) suggests that the overlayer structure formed at $1 \mathrm{ML}$ has to be very similar in these cases. Consequently, growth at $160 \mathrm{~K}$ and at $210 \mathrm{~K}$ follows different routes toward the same monolayer structure. We also note that a significantly lower monolayer $\triangle \mathrm{BE}$ value is found at $97 \mathrm{~K}(3.52 \mathrm{eV})$. A feasible explanation is that, at this temperature, second layer water adsorbs prior to the completion of the first layer. An observation supporting this notion is that the $\mathrm{O} 1 \mathrm{~s}$ intensity of the OD related component starts to decrease at this coverage (see Fig. 2), which can be due to signal attenuation from the second layer.

The most striking difference between the curves is the low $\triangle \mathrm{BE}$ values in the sub-ML regime observed upon growth at $210 \mathrm{~K}$. Generally, a decrease in the $\mathrm{O} 1 s \mathrm{BE}$ from water is expected upon the formation of hydrogen bonding networks. For water on the $\mathrm{TiO}_{2}(110)$ surface, aggregation within the first layer occurs by formation of $1 \mathrm{D}$ chains, with the expected result of a decrease in $\triangle \mathrm{BE}$ as the first layer is formed, as observed at 97 and $160 \mathrm{~K}$. For sub-ML coverage formed at $210 \mathrm{~K}$, however, the $\Delta \mathrm{BE}$ values are even lower than the value attained at $1 \mathrm{ML}$, which cannot simply be explained in terms of early formation of extended water aggregates. A critical property that differs growth at $210 \mathrm{~K}$ from growth at the two lower temperatures is the higher tendency for water dissociation at low coverage. As aggregates form molecular water will coordinate to hydroxyl groups formed by water dissociation [18]. The results in Fig. 3(b) therefore imply that the $\triangle \mathrm{BE}$ value for water coordinated to hydroxyls is lower than the average $\triangle \mathrm{BE}$ value of the full layer, which comprises of water bonded to water as well as hydroxyls.

The energetics of wetting of the $\mathrm{s}-\mathrm{TiO}_{2}$ surface has been studied earlier [18], but here we perform DFT calculations of both adsorption energies and $\mathrm{O} 1 s$ core-level BEs to gain a microscopic understanding and insight into the hydrogen bonding motifs as well as to probe their influences on the XPS spectra. Figure 4(a) shows a representative example of $30 \%$ coverage of water molecules on $\mathrm{TiO}_{2}(110)$ surface in three different configurations, namely $\mathbb{M} \rightarrow \mathbb{M} \rightarrow \mathbb{D}$, $\mathbb{M} \rightarrow \mathbb{M} \rightarrow \mathbb{M}$, and $\mathbb{M} \rightarrow \mathbb{D} \rightarrow \mathbb{M}$ (left to right). The arrow $(\rightarrow)$ indicates the direction of a donating hydrogen bond. The results for the adsorption energy versus coverage, summarized in Fig. 4(b), suggest that for low coverages $(<50 \%)$, the configurations with $\mathbb{M} \rightarrow \mathbb{D}$ are relatively more stable as compared to those with fully intact water molecules. However, the configurations containing $\mathbb{D} \rightarrow \mathbb{M}$ are least stable. Clearly, having a dissociated water molecule as a hydrogen bond donor (acceptor) is 
(a)

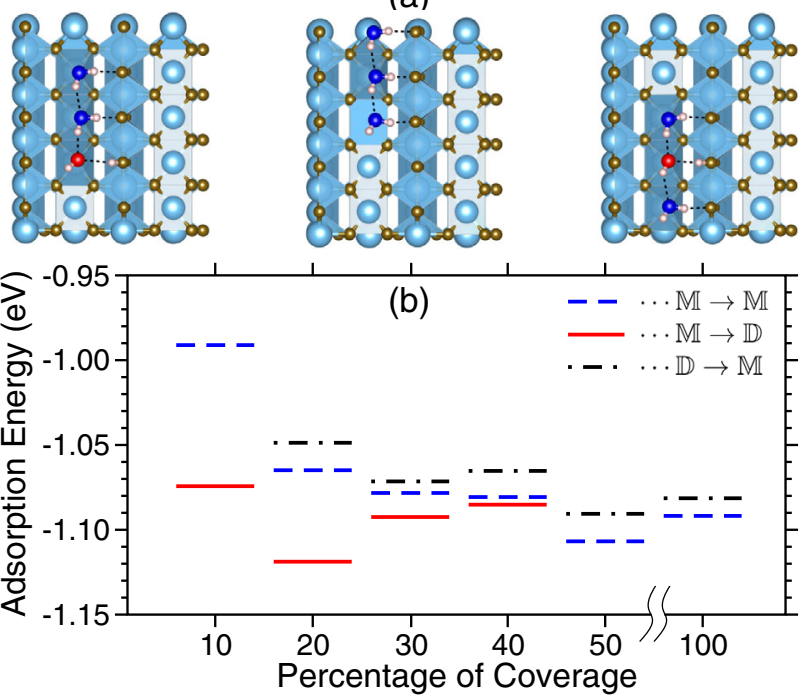

FIG. 4. (a) Top view of three possible geometric configurations $(\mathbb{M} \rightarrow \mathbb{D}, \mathbb{M} \rightarrow \mathbb{M}$, and $\mathbb{D} \rightarrow \mathbb{M})$ for $30 \%$ coverage of water molecules on $\mathrm{TiO}_{2}(110)$. (b) Adsorption energy versus coverage.

energetically unfavorable (favorable). Hence, for our models of higher coverages $50 \%$ and $100 \%$ in which rows are filled, the configurations with purely molecular water become energetically more favorable compared to those with a dissociated molecule.

We also calculated the $\Delta \mathrm{BE}$ for $\mathrm{D}_{2} \mathrm{O}$ on the $\mathrm{s}-\mathrm{TiO}_{2}$ surface for all adsorption models. To rationalize the $\triangle \mathrm{BE}$ of the $\mathrm{D}_{2} \mathrm{O}$ signal, we assess the influence of the local hydrogen bond environment $(X \rightarrow \mathbb{M} \rightarrow Y)$ for an intact water molecule (M), where as exemplified in Fig. 4(a), $X$ and $Y$ can be either empty $(\square), \mathbb{M}$, or $\mathbb{D}$. The $\Delta$ BEs for different coverages and local environments are summarized in Table I. See Fig. S2 in Supplemental Material for the geometrical configurations and their classification [40]. The accepting hydrogen bond (from $X$ ) has little influence on the $\triangle \mathrm{BE}$. Hence, XPS essentially probes three classes of intact water molecules with distinct
$\Delta$ BE; namely, (a) $X \rightarrow \mathbb{M} \rightarrow \square$, (b) $X \rightarrow \mathbb{M} \rightarrow \mathbb{M}$, and (c) $X \rightarrow \mathbb{M} \rightarrow \mathbb{D}$. It is clearly seen from Table I that the values of $\triangle \mathrm{BE}$ can be grouped into the three classes mentioned above. The highest and lowest values for $\triangle \mathrm{BE}$ are observed for configurations from classes (a) and (c), respectively, whereas the class (b) configurations yield intermediate values. Thus, these results clearly show that the acceptor $(Y)$ plays an important role in determining the BE. The decreasing $\triangle \mathrm{BE}$ can be explained by the enhanced stabilization of the core-ionized state due to the increasing electron rich hydrogen bond acceptors. In addition, the difference in $\mathrm{O} 1 \mathrm{~s} \mathrm{BE}$ for both the terminal and bridging hydroxyls is found to be small (about $0.18 \mathrm{eV}$ ), with the value of the former on the higher side. Moreover, the average $\mathrm{O} 1 s \mathrm{BE}$ of hydroxyls is about $2 \mathrm{eV}$ smaller than for molecular water. For evaluation, we also derived $\mathrm{O} 1 s \mathrm{BE}$ for $30 \%$ coverage using the $Z+1$ approximation with both a hybrid functional PBE0 [46] and PBE (see Supplemental Material [40]). The sensitivity in $\triangle \mathrm{BE}$ to $\mathrm{XC}$ functional is very small.

Our results on adsorption energy and $\triangle \mathrm{BE}$ clearly indicate that at $210 \mathrm{~K}$, predominantly class (c) type configurations are formed at low coverage (0.1 to $0.4 \mathrm{ML})$. In this regime, the energetically favorable configuration is $\mathbb{M} \rightarrow \mathbb{D}$ which belongs to class (c) that exhibits the lowest range of $\Delta \mathrm{BE}$. From 0.4 to $1 \mathrm{ML}$, the increasing values of $\Delta \mathrm{BE}$ and adsorption energy are consistent with formation of larger cluster sizes which comprise $\mathbb{M} \rightarrow \mathbb{M}$ configuration, belonging to class (b). At 97 and $160 \mathrm{~K}$, however, the dissociation probability is lower and, consistently, the values of $\triangle \mathrm{BE}$ suggest that environments of class (a) and class (b) dominate at low coverage. At monolayer coverage, the intermediate value of $\triangle \mathrm{BE}$ is due to configurations containing predominantly class (b) with a small contribution of class (a) environments at both 160 and $210 \mathrm{~K}$, whereas at $97 \mathrm{~K}$ it is likely that multilayer islands energetically preferred class (c) over class (a). That the uptake series at 160 and $210 \mathrm{~K}$ reach monolayer coverage through different routes can be

TABLE I. Difference in $\mathrm{BE}$ of $\mathrm{O} 1 s$ between intact $\mathrm{D}_{2} \mathrm{O}$ and $\mathrm{TiO}_{2}$, obtained from DFT based electronic structure calculations with the $\mathrm{PBE}$ functional, versus coverage for various hydrogen bonding configurations. The last column contains the overall range of $\Delta \mathrm{BE}$ values obtained for the classes.

\begin{tabular}{|c|c|c|c|c|c|c|c|c|}
\hline \multirow[t]{2}{*}{ Class } & \multicolumn{4}{|l|}{ Configuration } & \multicolumn{4}{|l|}{$\Delta \mathrm{BE}(\mathrm{eV})$} \\
\hline & $X \rightarrow \mathbb{M} \rightarrow Y$ & $10 \%$ & $20 \%$ & $30 \%$ & $40 \%$ & $50 \%$ & $100 \%$ & Range \\
\hline \multirow[t]{3}{*}{$\mathrm{a}$} & $\mathbb{M} \rightarrow \mathbb{M} \cdots \square$ & & 4.07 & 3.96 & 3.96 & & & \multirow{3}{*}{$3.91-4.07$} \\
\hline & $\mathbb{D} \rightarrow \mathbb{M} \cdots \square$ & & & 3.91 & 3.92 & & & \\
\hline & $\square \cdots \mathbb{M} \cdots \square$ & 3.97 & & & & & & \\
\hline \multirow[t]{3}{*}{$\mathrm{b}$} & $\mathbb{M} \rightarrow \mathbb{M} \rightarrow \mathbb{M}$ & & & 3.41 & $3.40-3.61$ & $3.49-3.58$ & $3.45-3.51$ & \multirow{3}{*}{$3.36-3.61$} \\
\hline & $\mathbb{D} \rightarrow \mathbb{M} \rightarrow \mathbb{M}$ & & & & & 3.44 & 3.37 & \\
\hline & $\square \cdots \mathbb{M} \rightarrow \mathbb{M}$ & & 3.36 & $3.36-3.43$ & $3.36-3.39$ & & & \\
\hline \multirow[t]{2}{*}{$\mathrm{c}$} & $\mathbb{M} \rightarrow \mathbb{M} \rightarrow \mathbb{D}$ & & & 3.11 & $3.11-3.22$ & 3.24 & $3.16-3.17$ & \multirow{2}{*}{$2.93-3.24$} \\
\hline & $\square \cdots \mathbb{M} \rightarrow \mathbb{D}$ & & 2.93 & 3.08 & & & & \\
\hline
\end{tabular}


rationalized in terms of surface diffusion effects. Diffusion along the $\mathrm{Ti}_{5 \mathrm{c}}$ rows at $210 \mathrm{~K}$ is more than 1000 times faster than at $160 \mathrm{~K}$ [24]. However, if the temperature is too low to permit appreciable diffusion along the $\mathrm{Ti}_{5 c}$ row, transient diffusion along the $\mathrm{O}_{b}$ rows or on top of already adsorbed water can occur before the molecule adsorbs on a $\mathrm{Ti}_{5 c}$ site. The different kinetics involved in nucleation and aggregation can thus yield metastable low-coverage configurations at $160 \mathrm{~K}$ (and $97 \mathrm{~K}$ ) analogous to the behavior found for water on anatase $\mathrm{TiO}_{2}(101)$ [47].

In conclusion, measurements and simulations of $\mathrm{O} 1 \mathrm{~s}$ XPS for water on the stoichiometric $\mathrm{TiO}_{2}(110)$ surface allow for insight into the mechanism of formation of first water layer as well as the temperature dependent kinetics of intact $\left(\mathrm{D}_{2} \mathrm{O}\right)$ and dissociated (OD) water as a function of coverage and temperature. The uptake series at 160 and $210 \mathrm{~K}$ reach monolayer coverage through different routes, with higher degree of dissociation at $210 \mathrm{~K}$, associated with lower values of $\triangle \mathrm{BE}$ for intact water in the submonolayer regime. Palpable differences in $\triangle \mathrm{BE}$ versus coverage can be rationalized in terms of three distinct classes based on the local hydrogen bonding arrangement around intact water. The above findings will lead to further advancement in molecular-level understanding of water's role in several key areas such as electrocatalysis, heterogeneous catalysis, photoelectrochemical water splitting, as well as in the renewable water-oxide energy interface technologies [48-50].

This work was supported by the Swedish Research Council (VR Contract No. 2015-03956) and the Swedish energy agency (Contract No. 2017-006797). The calculations were performed on resources provided by the Swedish National Infrastructure for Computing (SNIC).

*ckamal@rrcat.gov.in

†odelius@fysik.su.se

*anders.sandell@physics.uu.se

[1] V. E. Henrich and P. A. Cox, The Surface Science of Metal Oxides (Cambridge University Press, Cambridge, England, 1994).

[2] G. E. Brown, Science 294, 67 (2001).

[3] C. Lun Pang, R. Lindsay, and G. Thornton, Chem. Soc. Rev. 37, 2328 (2008).

[4] Z. Dohnálek, I. Lyubinetsky, and R. Rousseau, Prog. Surf. Sci. 85, 161 (2010).

[5] H. H. Kristoffersen, J. O. Hansen, U. Martinez, Y. Y. Wei, J. Matthiesen, R. Streber, R. Bechstein, E. Lægsgaard, F. Besenbacher, B. Hammer et al., Phys. Rev. Lett. 110, 146101 (2013).

[6] G. E. Ewing, Chem. Rev. 106, 1511 (2006).

[7] G. Ketteler, S. Yamamoto, H. Bluhm, K. Andersson, D. E. Starr, D. F. Ogletree, H. Ogasawara, A. Nilsson, and M. Salmeron, J. Phys. Chem. C 111, 8278 (2007).

[8] J. T. Newberg, D. E. Starr, S. Yamamoto, S. Kaya, T. Kendelewicz, E. R. Mysak, S. Porsgaard, M. B. Salmeron,
G. E. Brown, A. Nilsson et al., J. Phys. Chem. C 115, 12864 (2011).

[9] M. Gratzel, Nature (London) 414, 338 (2001).

[10] M. A. Henderson, Surf. Sci. Rep. 46, 1 (2002).

[11] U. Diebold, Surf. Sci. Rep. 48, 53 (2003).

[12] C. L. Pang, R. Lindsay, and G. Thornton, Chem. Rev. 113, 3887 (2013).

[13] P. M. Kowalski, B. Meyer, and D. Marx, Phys. Rev. B 79, 115410 (2009).

[14] L.-M. Liu, C. Zhang, G. Thornton, and A. Michaelides, Phys. Rev. B 82, 161415(R) (2010).

[15] L. E. Walle, A. Borg, P. Uvdal, and A. Sandell, Phys. Rev. B 80, 235436 (2009).

[16] L. Walle, D. Ragazzon, A. Borg, P. Uvdal, and A. Sandell, Surf. Sci. 621, 77 (2014).

[17] D. A. Duncan, F. Allegretti, and D. P. Woodruff, Phys. Rev. B 86, 045411 (2012).

[18] M. Amft, L. E. Walle, D. Ragazzon, A. Borg, P. Uvdal, N. V. Skorodumova, and A. Sandell, J. Phys. Chem. C 117, 17078 (2013).

[19] Y. Du, N. A. Deskins, Z. Zhang, Z. Dohnálek, M. Dupuis, and I. Lyubinetsky, Phys. Rev. Lett. 102, 096102 (2009).

[20] K. R. Hahn, A. Tricoli, G. Santarossa, A. Vargas, and A. Baiker, Langmuir 28, 1646 (2012).

[21] K. Sebbari, C. Domain, J. Roques, H. Perron, E. Simoni, and H. Catalette, Surf. Sci. 605, 1275 (2011).

[22] I. M. Brookes, C. A. Muryn, and G. Thornton, Phys. Rev. Lett. 87, 266103 (2001).

[23] G. Teobaldi, W. Hofer, O. Bikondoa, C. Pang, G. Cabailh, and G. Thornton, Chem. Phys. Lett. 437, 73 (2007).

[24] J. Matthiesen, J. O. Hansen, S. Wendt, E. Lira, R. Schaub, E. Lægsgaard, F. Besenbacher, and B. Hammer, Phys. Rev. Lett. 102, 226101 (2009).

[25] Z.-T. Wang, Y.-G. Wang, R. Mu, Y. Yoon, A. Dahal, G. K. Schenter, V.-A. Glezakou, R. Rousseau, I. Lyubinetsky, and Z. Dohnálek, Proc. Natl. Acad. Sci. U.S.A. 114, 1801 (2017).

[26] J. Andersen, O. Björneholm, A. Sandell, R. Nyholm, J. Forsell, L. Thånell, A. Nilsson, and N. Mårtensson, Synchrotron Radiat. News 4, 15 (1991).

[27] M. A. Henderson, Langmuir 12, 5093 (1996).

[28] S. Wendt, P. T. Sprunger, E. Lira, G. K. H. Madsen, Z. Li, J. Ø. Hansen, J. Matthiesen, A. Blekinge-Rasmussen, E. Lægsgaard, B. Hammer et al., Science 320, 1755 (2008).

[29] L. E. Walle, A. Borg, P. Uvdal, and A. Sandell, Phys. Rev. B 86, 205415 (2012).

[30] Z. Zhang, Y. Du, N. G. Petrik, G. A. Kimmel, I. Lyubinetsky, and Z. Dohnálek, J. Phys. Chem. C 113, 1908 (2009).

[31] K. Andersson, A. Nikitin, L. G. M. Pettersson, A. Nilsson, and H. Ogasawara, Phys. Rev. Lett. 93, 196101 (2004).

[32] L. E. Walle, D. Ragazzon, A. Borg, P. Uvdal, and A. Sandell, Appl. Surf. Sci. 303, 245 (2014).

[33] J. Hutter, M. Iannuzzi, F. Schiffmann, and J. VandeVondele, WIREs Comput. Mol. Sci. 4, 15 (2014).

[34] J. P. Perdew, K. Burke, and M. Ernzerhof, Phys. Rev. Lett. 77, 3865 (1996).

[35] S. Grimme, J. Antony, S. Ehrlich, and H. Krieg, J. Chem. Phys. 132, 154104 (2010).

[36] G. Lippert, J. Hutter, and M. Parrinello, Mol. Phys. 92, 477 (1997), https://www.tandfonline.com/doi/abs/10.1080/ 002689797170220. 
[37] M. Krack and M. Parrinello, Phys. Chem. Chem. Phys. 2, 2105 (2000).

[38] C. E. Patrick and F. Giustino, Phys. Rev. Applied 2, 014001 (2014).

[39] C. E. Patrick and F. Giustino, Phys. Rev. B 84, 085330 (2011).

[40] See Supplemental Material at http://link.aps.org/supplemental/ 10.1103/PhysRevLett.126.016102 for more details for (a) the clean $\mathrm{TiO}_{2}(110)$ surface, (b) the geometrical configurations and their classification, and (c) influence of XC functional on $\triangle \mathrm{BE}$, which also includes Refs. [41-45].

[41] M. J. Jackman, A. G. Thomas, and C. Muryn, J. Phys. Chem. C 119, 13682 (2015).

[42] J. O. Nilsson, M. Leetmaa, B. Wang, P. A. Žguns, I. Pašti, A. Sandell, and N. V. Skorodumova, Phys. Status Solidi B 255, 1700344 (2018).
[43] F. Labat, P. Baranek, C. Domain, C. Minot, and C. Adamo, J. Chem. Phys. 126, 154703 (2007).

[44] S. C. Ammal and A. Heyden, J. Chem. Phys. 133, 164703 (2010).

[45] V. Vetere, C. Adamo, and P. Maldivi, Chem. Phys. Lett. 325, 99 (2000).

[46] C. Adamo and V. Barone, J. Chem. Phys. 110, 6158 (1999).

[47] A. Dahal and Z. Dohnálek, J. Phys. Chem. C 121, 20413 (2017).

[48] C. Ros, T. Andreu, and J. R. Morante, J. Mater. Chem. A 8, 10625 (2020).

[49] V. R. Stamenkovic, D. Strmcnik, P. P. Lopes, and N. M. Markovic, Nat. Mater 16, 57 (2017).

[50] I. Katsounaros, S. Cherevko, A. R. Zeradjanin, and K. J. J. Mayrhofer, Angew. Chem., Int. Ed. Engl. 53, 102 (2014). 